\title{
Molecular packing in double gyroid cubic phases revealed via resonant soft X-ray scattering \\ Chenhui Zhu ${ }^{1}$ \\ ${ }^{1}$ Lawrence Berkeley National Laboratory \\ chenhuizhu@lbl.gov
}

The bicontinuous double gyroid phase is one of the nature's most symmetric and complex structures, the electron density map of which was established long ago. By utilizing small-angle X-ray scattering (SAXS), resonant soft Xray scattering (RSoXS) at the carbon K-edge and model-dependent tensor based scattering theory, we have not only elucidated morphology but also identified molecular packing in the double gyroid phases formed by molecules with different shapes, i.e. rod-like vs taper-shaped, thus validating some of the hypothetical packing models and disproving others. The spatial variation of molecular orientation through the channel junctions in the double gyroid phase can be either continuous in the case of anisotropic channels or discontinuous in the case of isotropic channels depending on the molecular structure and shape.

*main ref: Yu Cao, Mohamed Alaasar, Asritha Nallapaneni, Mirosław Salamończyk, Peter Marinko, Ewa Gorecka, Carsten Tschierske, Feng Liu, Nataša Vaupotič, Chenhui Zhu, Phys. Rev. Lett. 125, 027801 (2020)

*We acknowledge the Advanced Light Source supported by the Director of the Office of Science, Office of Basic Energy Sciences, of the U.S. Department of Energy under contract no. DE-AC02-05CH11231, National Natural Science Foundation of China (No. 21761132033 and 21774099), Science and Technology Agency of Shaanxi Province (No. 2016KW-050 and 2018KWZ-03), National Science Centre (Poland) no. 2016/22/A/ST5/00319, Slovenian Research Agency-no. P1-0055. 